\title{
Glaukommedikation: signifikante Assoziation zwischen Non-Adhärenz und Sehverlust
}

\author{
Newman-Casey PA et al. The Association between \\ Medication Adherence and Visual Field Progres- \\ sion in the Collaborative Initial Glaucoma Treat- \\ ment Study. Ophthalmol 2020; 127: 477-483
}

Beim Glaukom kommt die medikamentöse Therapie am häufigsten zum Einsatz. Schätzungen zufolge liegt die Therapietreue in den USA aber nur bei etwa $50 \%$ und nimmt mit zunehmender Behandlungsdauer weiter ab. Auch wenn es naheliegend erscheinen mag, dass eine schlechte Medikamentenadhärenz zu schlechten Therapieergebnissen führt, bleibt die Frage nach der Wirksamkeit bei zumindest partieller Adhärenz.

In der multizentrischen, randomisierten „Collaborative Initial Glaucoma Treatment Study“ (CIGTS) ging es um die Frage, ob eine initiale medikamentöse Glaukomtherapie genauso effektiv ist wie ein primäre Operation (Trabekulektomie). Fast 80\% der CIGTS-Teilnehmer nahmen über den gesamten Nachbeobachtungszeitraum von 5 Jahren an der Studie teil. Somit bietet CIGTS die einzigartige Möglichkeit, den Zusammenhang zwischen Einnahmeverhalten und Glaukomprogression zu un- 
tersuchen. Paula Anne Newman-Casey und Kollegen stellen aktuell ihre Ergebnisse dazu vor.

In der CIGTS wurden 607 neu diagnostizierte Patienten mit Offenwinkelglaukom randomisiert auf beide Gruppen verteilt und entsprechend behandelt. Die Nachuntersuchungen erfolgten alle 6 Monate und erstreckten sich auf bis zu 10 Jahre. Erfasst wurden dabei die Gesichtsfeldentwicklung, die Lebensqualität und die Medikamentenadhärenz. Deren Abfrage erfolgte mittels Telefoninterviews („Haben Sie gestern eine Dosis Ihrer Medikamente vergessen?").

Für die vorliegende Studie wurden die Daten aller 307 Patienten im Medikamentenarm der CIGTS ausgewertet. Ihr durchschnittliches Alter lag bei 57 Jahren, die durchschnittliche Nachbeobachtungszeit bei 7,3 Jahren.
Nimmt man alle Nachuntersuchungen zusammen, gaben $46 \%$ der Patienten $(n=$ 143) an, dass sie niemals eine Dosis ihres Glaukommedikaments vergessen hatten, $37 \%$ ( $n=112$ ) berichteten bei bis zu einem Drittel der Befragungen über eine verpasste Dosis, $10 \%(n=31)$ gaben bei einem bis zwei Drittel der Befragungen eine verpasste Dosis an und $7 \%(n=21)$ gaben bei über zwei Drittel der Befragungen eine verpasste Dosis an. Patienten mit schlechter Medikamentenadhärenz waren im Vergleich zu Patienten ohne verpasste Dosis durchschnittlich 10 Jahre jünger, nicht verheiratet und von schwarzer Ethnizität, und sie hatten zu Beginn größere Gesichtsfeldschäden, eine schlechtere Schulbildung und eine höhere Punktzahl in einem Depressionsfragebogen.

Eine höhere Anzahl an Befragungen, bei denen ein Patient eine verpasste Medikamentendosis angab, war signifikant assozi- iert mit einer Abnahme (Verschlechterung) der mittleren Abweichung (MD) im Gesichtsfeld (geschätzte $-0,14 \mathrm{~dB}$ pro Besuch mit verpasster Dosis). Bei einem Patienten, der niemals eine Medikamentendosis verpasste, betrug die durchschnittliche prognostizierte Verschlechterung der MD über 8 Jahre $-0,62 \mathrm{~dB}$, was mit Werten des altersbedingten Verlusts bei stabilem Glaukom übereinstimmt. Bei einem Patienten, der bei bis zu einem Drittel der Befragungen eine verpasste Dosis angab, lag der Wert bei $-1,42 \mathrm{~dB}$, bei einem Patienten, der bei einem bis zwei Drittel der Befragungen eine verpasste Dosis hatte, bei $-1,82 \mathrm{~dB}$ und bei einem Patienten, der bei über zwei Drittel der Befragungen eine verpasste Dosis angab, bei $-2,23 \mathrm{~dB}$.

\section{FAZIT}

Die Auswertung der CIGTS-Studie ergab eine statistisch und klinisch signifikante Assoziation zwischen der Medikamentenadhärenz von Patienten mit Offenwinkelglaukom und dem glaukombedingten Sehverlust. Die Daten zeigten eine eindeutige Dosis-Wirkungs-Beziehung auf. Pluspunkt der Studie ist der lange Beobachtungszeitraum von etwa 8 Jahren. Außerdem konnten mehrere Faktoren identifiziert werden, die zur schlechten Adhärenz beizutragen scheinen.

Dr. Michaela Bitzer, Tübingen 